\title{
Spot the Difference: A Case of Hailey-Hailey Disease in a 64-year-old Filipino Female
}

\author{
Raisa Celine R. Rosete, MD, MBA, Juan Paolo David S. Villena, MD and Mae N. Ramirez-Quizon, MD \\ Department of Dermatology, Philippine General Hospital, University of the Philippines Manila
}

\begin{abstract}
Hailey-Hailey disease (HHD) is an uncommon acantholytic disorder of the skin. This is a case of a 64-year-old Filipino female with a chronic history of painful and malodorous intertriginous plaques. Histopathologic evaluation showed overlapping features of pemphigus vulgaris and Hailey-Hailey disease. A negative direct immunofluorescence test clinched the diagnosis of Hailey-Hailey disease. The patient was advised regarding preventive measures and treated with topical antibiotics and corticosteroids with improvement of her lesions.
\end{abstract}

Key Words: Hailey-Hailey Disease, Chronic Benign Familial Pemphigus, Direct Immunofluorescence

\section{INTRODUCTION}

Hailey-Hailey disease (HHD) or chronic benign familial pemphigus is an uncommon autosomal dominant acantholytic disorder of the skin that presents as crusted, weeping, annular plaques at intertriginous sites of the body. No gender or ethnic group predilection is noted and it usually presents at the second to fourth decades of life. ${ }^{1}$ Diagnosis of HHD is based on lesion morphology and location, family history, and the characteristic dilapidated brick wall appearance of the epidermis on histopathologic evaluation. ${ }^{2}$ Historically, HHD and pemphigus vulgaris were thought to be related due to their histologic similarities and only with the advent of immunofluorescence studies were they categorized as independent entities. ${ }^{3}$ Based on Philippine data, only 23 cases of Hailey-Hailey disease have been recorded in the past 8 years. ${ }^{4}$ In this report, we present a case of late-onset Hailey-Hailey disease in a 64-year old Filipino woman with overlapping histopathologic features with pemphigus vulgaris/vegetans that required direct immunofluorescence for final diagnostic confirmation.

\section{CASE DESCRIPTION}

This is a case of MM, a 64-year-old Filipino female, who presented with a two-year history of multiple, pruritic, foul-smelling erythematous to violaceous weeping plaques that initially presented as multiple vesicles and pustules, distributed over her anterior neck folds, axillae, antecubital fossae, inframammary area, and inguinal area. There was no associated mucosal involvement. The lesions were painful and malodorous and worsened with sweating and excessive friction. Her mother, daughter, son, and female grandchild

exhibited similar lesions.

Department of Dermatology

Philippine General Hospital

University of the Philippines Manila

Taft Avenue, Ermita, Manila 1000, Philippines

Email:: rrrosete@up.edu.ph 
The lesions were recurrent, noted to flare approximately four to five times a year. The patient consulted with multiple physicians and was managed as a case of eczema or tinea, prescribed with different combinations of topical steroids and antifungals with only minimal to partial resolution of her symptoms. The patient was then referred to our institution for further evaluation and management.

On physical examination, the lesions were symmetrically distributed over the intertriginous areas of the skin (Figure 1A). Multiple, well-demarcated erythematous annular plaques with overlying whitish scaling and hemorrhagic crusting were seen on the anterior neck folds and bilateral antecubital fossae, with no evidence of vesicles, pustules, or erosions (Figures 1B, 1C).

Thicker erythematous to violaceous, well-demarcated, macerated plaques with overlying erosions with bright-red bases were seen over the inframammary area, inguinal area, and bilateral axillary area, still with no evidence of vesicles or pustules (Figures $2 \mathrm{~A}, 2 \mathrm{~B}, 2 \mathrm{C}$ ).
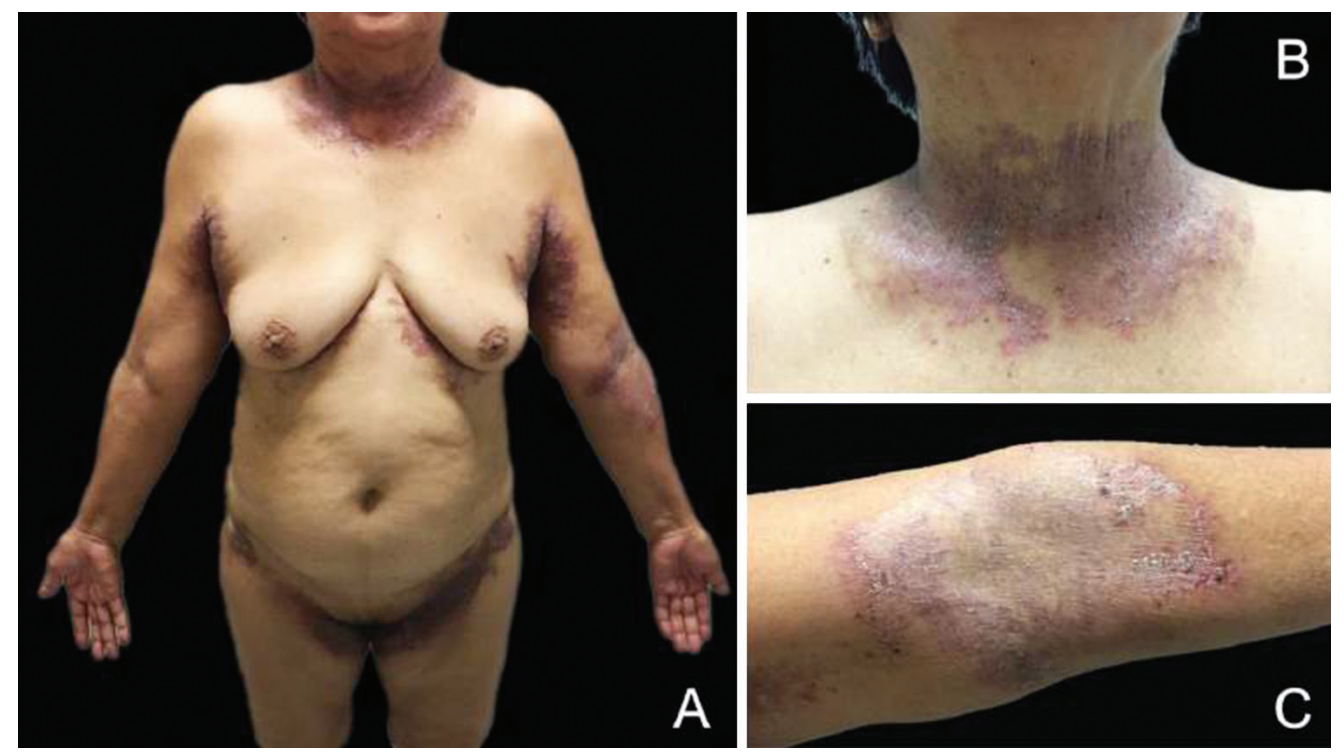

Figure 1. Clinical Findings of Hailey-Hailey Disease. Multiple, erythematous, annular, scaly plaques seen in primarily intertriginous sites (A), with involvement of the anterior folds of the neck (B) and antecubital fossae (C).
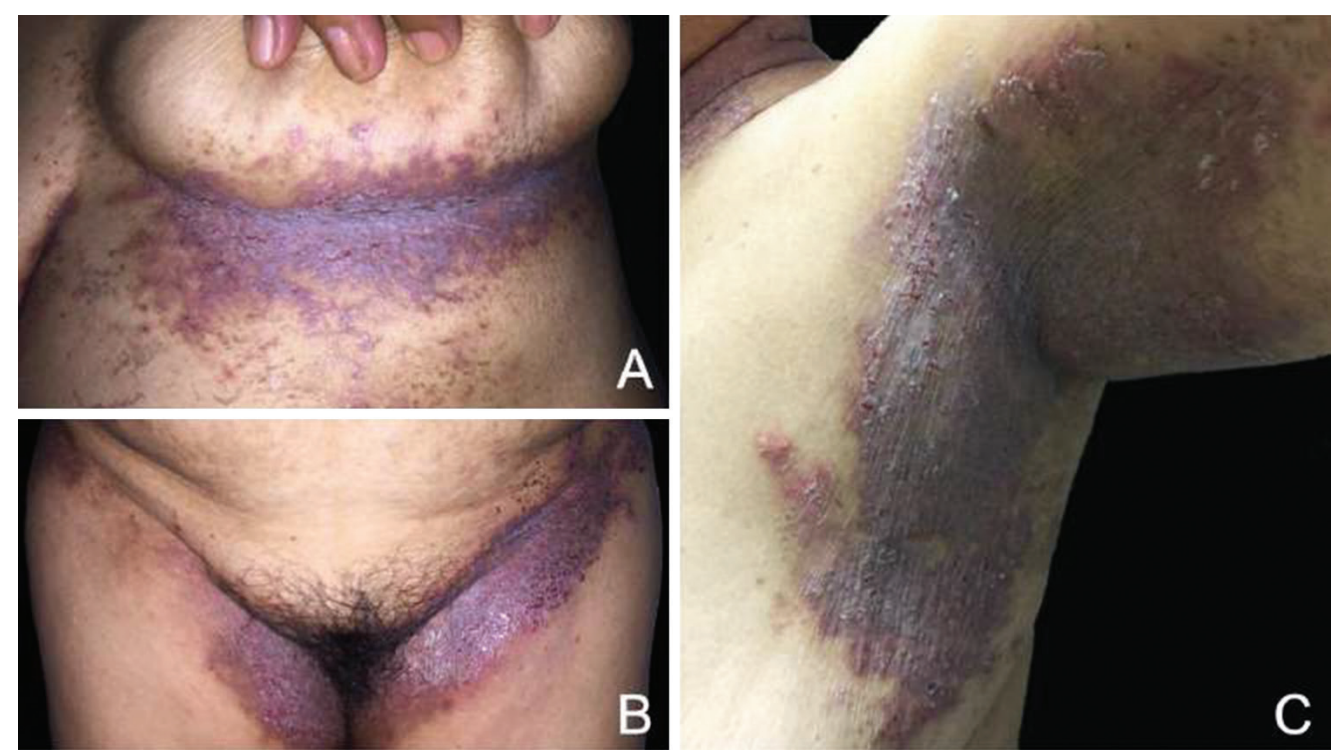

Figure 2. Clinical Findings of Hailey-Hailey Disease. Thick, macerated, weeping erythematous to violaceous plaques with red-based erosions over the inframammary area (A), inguinal area (B), and axillary area (C). 


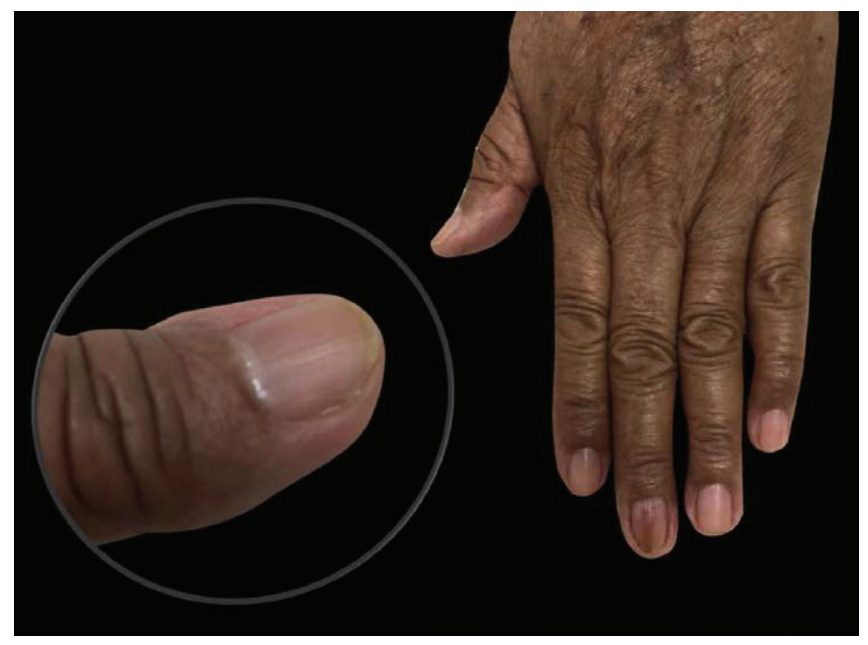

Figure 3. Clinical Findings of Hailey-Hailey Disease. Longitudinal white bands (longitudinal leukonychia) of the nails, seen more prominently on the fingernails of this patient.

Longitudinal leukonychia (faint, longitudinal white bands on the nails) was appreciated on examination of the nails, more prominently seen over the fingernails (Figure 3).

Wood's lamp was negative for coral-red fluorescence and $\mathrm{KOH}$ preparation was negative for hyphal, pseudohyphal, and yeast elements. Two 4-mm punch biopsies were then taken from the left antecubital fossa and from the left inframammary area. The biopsy from the left antecubital fossa showed intraepithelial splitting, with the presence of rounded-off, eosinophilic acantholytic cells within the cleft (Figure 4A, 4C). A single layer of intact basal cells or the tombstoning pattern is also seen (Figure $4 \mathrm{C}$ ). In the dermis, moderate inflammatory infiltrates of neutrophils and conspicuous eosinophils were appreciated (Figure 4B).

The biopsy taken from the left inframammary area still showed areas of intraepithelial splitting with a single layer of intact basal cells or the tombstoning pattern (Figure $5 \mathrm{~A})$, however there are areas that exhibit only partial loss of cohesion between keratinocytes, with an appearance likened to a dilapidated brick wall (Figure 5B, 5C).

Direct immunofluorescence (DIF) was performed, which showed negative $\operatorname{IgG}, \operatorname{IgM}, \operatorname{IgA}$, and C3 deposition on all slides. The clinical presentation, positive family history, and combined histopathologic and DIF findings were consistent with Hailey-Hailey disease or chronic benign familial pemphigus. The patient was advised weight reduction, the use of light-weight and loose clothing, as well as mupirocin $2 \%$ ointment twice a day for 7 days, triamcinolone acetonide $0.1 \%$ lotion twice a day for 14 days and chlorphenamine maleate $4 \mathrm{mg}$ once a day at night to alleviate night-time pruritus.

On follow-up after 2 weeks, there was marked decrease in the erythema, pruritus, foul-smelling odor over all the affected areas (Figure 6).

\section{DISCUSSION}

Hailey-Hailey disease or chronic benign familial pemphigus is an autosomal dominant acantholytic disorder of the skin. It is caused by mutations in the gene ATP2C1, which codes for an ATP-powered calcium pump
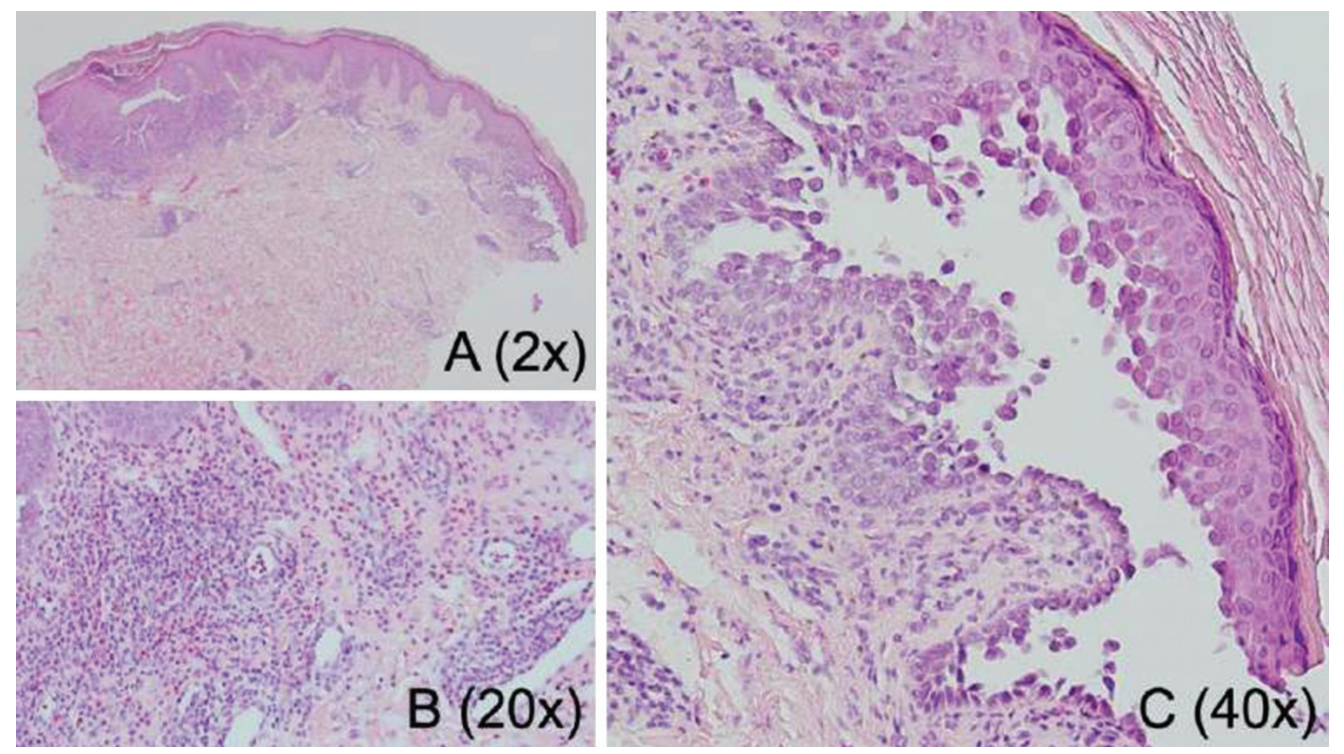

Figure 4. Histopathologic review of 4mm-punch biopsy taken from the left antecubital fossa (Hematoxylin and Eosin). These sections show intraepithelial splitting, acantholytic cells, and the tombstoning pattern. The dermis shows neutrophilic infiltrates with numerous eosinophils. 
on the Golgi membrane - the human secretory pathway $\mathrm{Ca}^{2+} / \mathrm{Mn}^{2+}$ ATPase isoform 1 or SPCA1 protein. Defects in this intercellular calcium pump disrupts calcium homeostasis, which affect different processes such as cellular differentiation, cellular adhesion, and keratinocyte mobility. Changes in calcium levels lead to alteration of desmosomes and the cellular adhesion of keratinocytes to the epidermal junction, which will eventually cause acantholysis. ${ }^{5}$

Hailey-Hailey disease has an incidence of 1 in 50,000, which makes it a fairly uncommon condition worldwide.
In the Philippines, only 23 cases were reported in the past 8 years. ${ }^{4}$ No gender predilection is noted, and the disease primarily presents within the second and fourth decades of life. ${ }^{1}$ This case is unique as the patient presented with late-onset HHD ( $6^{\text {th }}$ decade of life), in comparison to her daughter, son and female grandchild, who all presented at younger ages. A similar case of late-onset Hailey-Hailey disease was reported in 2019, presenting with involvement of the non-intertriginous regions. Severity of disease also varied between family members, the son presenting with
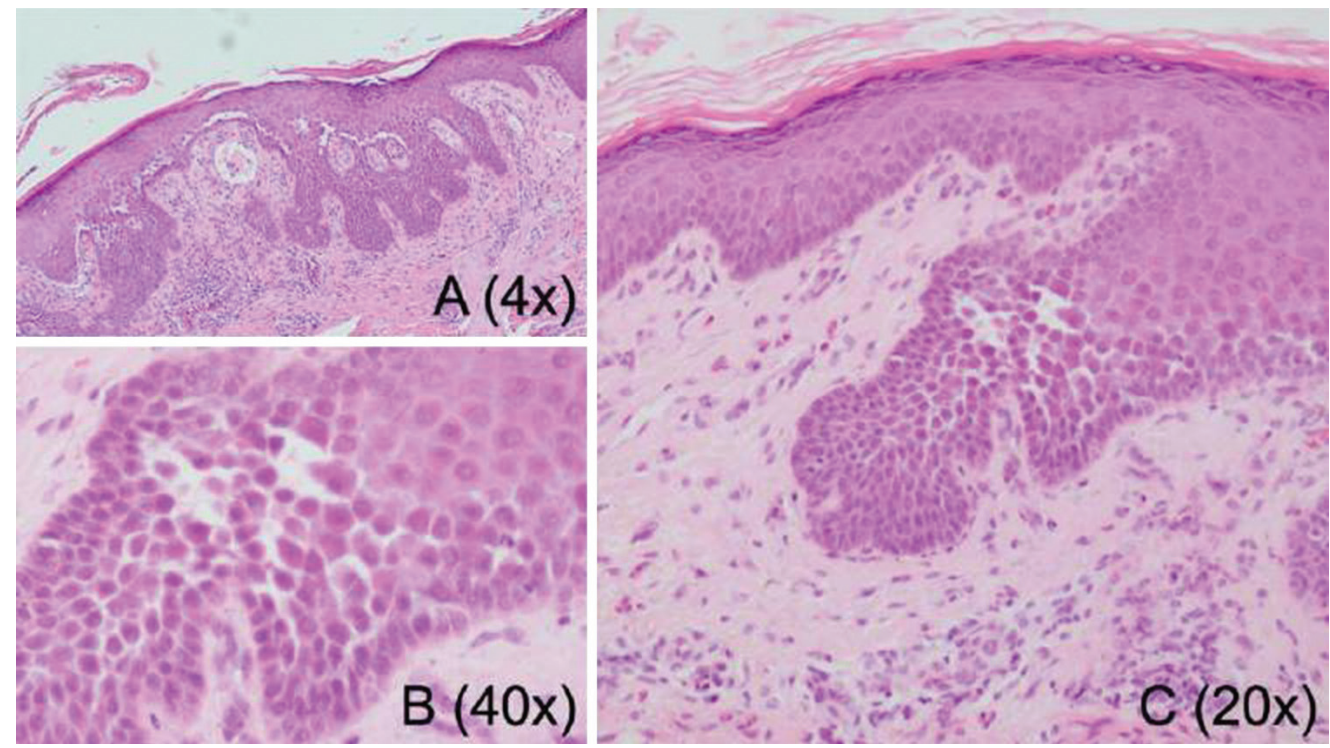

Figure 5. Histopathologic review of 4mm-punch biopsy taken from the left inframammary area (Hematoxylin and Eosin). These sections show intraepithelial splitting with a tombstoning pattern, and the dilapidated brick wall pattern in some areas.

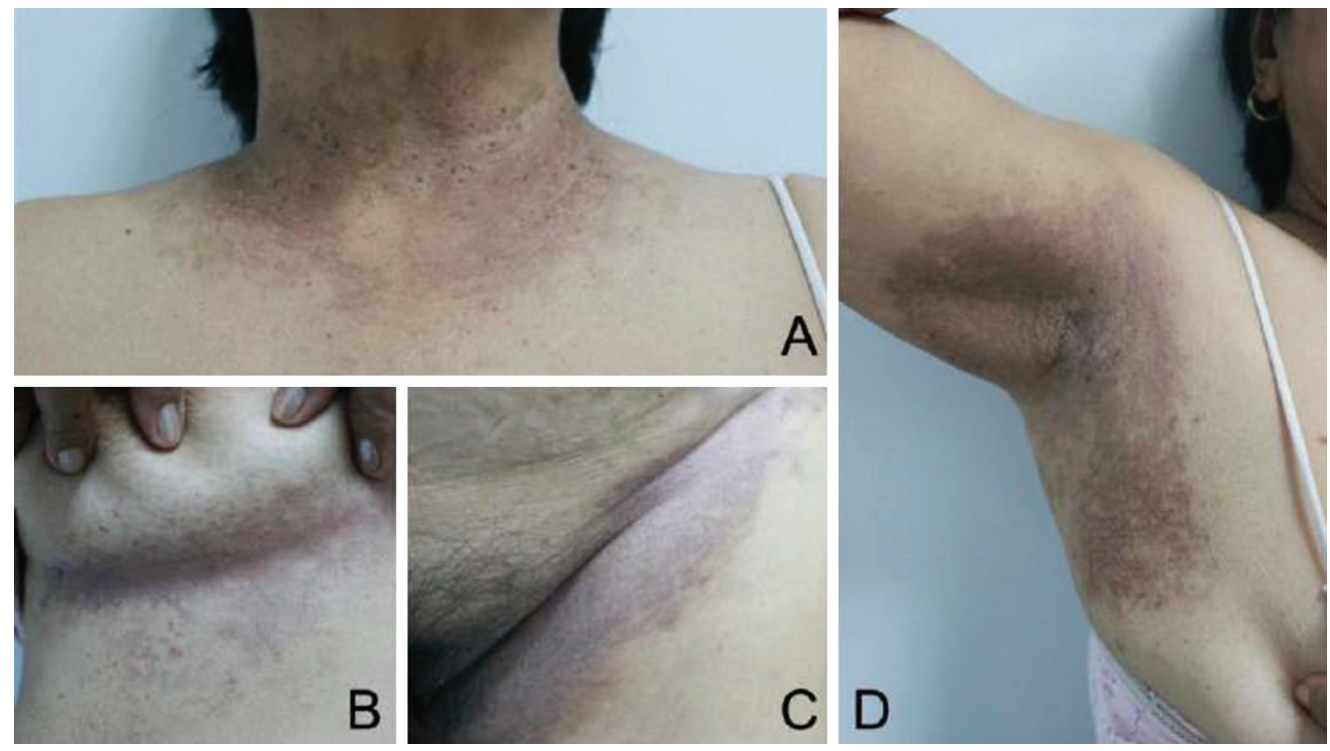

Figure 6. Hailey-Hailey disease after 2 weeks of treatment with topical antimicrobials and topical steroids. 
milder lesions and fewer relapses. Variation in clinical manifestations can be attributed to intrinsic as well as extrinsic factors. A study conducted by Beek et al. in 2015 concluded that levels of other ATPase proteins may influence expressivity of HHD disease. Additional mutations in these genes may result in certain individuals being more vulnerable to environmental triggers ${ }^{6}$, which could be a possible factor in the late-onset of this disease in our patient. Studies also suggested that the severity of clinical phenotype is dictated by the severity of SPCA1 impairment, which can explain the variation between the different family members of our patient. In addition to these intrinsic genetic factors, extrinsic factors that could also affect presentation include high temperature, minor trauma, and pathogens. ${ }^{7}$

The diagnosis of HHD is often delayed as its clinical presentation often resembles common dermatoses. In this case, our patient consulted multiple times with different physicians due to recurrent symptoms, and she was managed as either eczema or tinea and was prescribed different steroids and antifungal creams with varying results. Delayed diagnosis, coupled with the pain, pruritus, and malodor of the disease can greatly impact the quality of life of these patients. DLQI scores of patients with HaileyHailey disease were reported to be $20.2 \%$ of the maximum score, comparable to the $29.7 \%$ score in psoriasis. A more recent study used Skindex-29 and median quality of life scores of the patients studied were greater than 35 which signified very poor quality of life. ${ }^{8}$ Moreover, the general prognosis of this disease is also relatively poor, as it has a chronic course with multiple relapses, though in some patients it is shown to improve with older age. ${ }^{1}$

In addition to mimicking common dermatoses clinically, Hailey-Hailey disease shares histopathological features with pemphigus vulgaris or pemphigus vegetans. As such, these were considered related disease entities historically. ${ }^{3}$

With the intertriginous distribution of the lesions, the histopathological features of intraepithelial splitting with a tombstone pattern, as well as neutrophilic and eosinophilic infiltrates, pemphigus vegetans, a rare variant of pemphigus vulgaris, was another differential considered for this case. Pemphigus vegetans however more commonly presents with thicker, vegetative plaques as well as mucosal lesions. Findings consistent with a diagnosis of HaileyHailey disease include the intertriginous distribution of the lesions, the familial involvement, as well as areas that resemble the characteristic dilapidated brick wall pattern on histopathology. However, the neutrophilic and eosinophilic infiltrates found on histopathologic evaluation were not consistent with a picture of Hailey-Hailey disease. Clinical considerations on initial evaluation of this case included an early presentation of non-mucosal pemphigus vegetans. While mucosal involvement is characteristic of pemphigus vegetans, isolated cases without mucosal involvement have been reported throughout literature. ${ }^{9}$ A more likely scenario however was that this was a case of Hailey-Hailey disease with chronic lesions and a possible concomitant allergic contact dermatitis, which could explain the considerable eosinophilic infiltrates found on histopathology. In addition to this, though the patient denies any contactants, contact dermatitis has also been shown to trigger or exacerbate cases of HHD. ${ }^{1}$

The advent of immunofluorescence studies allowed for the differentiation of Hailey-Hailey disease and pemphigus vulgaris and vegetans. ${ }^{3}$ Pemphigus vulgaris and pemphigus vegetans often present with IgG and $\mathrm{C} 3$ deposition in the epidermal intercellular space in $80-90 \%$ of cases, according to a study conducted by Arbache et al. in 2014 . In comparison, Hailey-Hailey disease usually presents with negative DIF studies. ${ }^{10}$ Direct immunofluorescence was performed, which showed negative $\operatorname{IgG}, \operatorname{IgM}, \operatorname{IgA}$, and $\mathrm{C} 3$ deposition on all slides. This led to the final diagnosis of Hailey-Hailey disease for this patient.

As Hailey-Hailey disease is a relatively uncommon genetic dermatosis, there is a lack of evidence-based guidelines for its treatment. In addition to this, the chronicity and tendency for relapse in this disease makes HHD challenging to manage for physicians and dermatologists alike. Proven first-line treatments include use of antiseptics and topical or oral antimicrobials, as superimposed bacterial or candidal infections are common and these can aggravate lesions and impede healing. Topical corticosteroids are considered first-line therapeutic agents and are used to control skin inflammation. However, they are only reserved for acute flares, as prolonged treatment with corticosteroids can predispose the patient to secondary infections. Nonpharmacologic therapy includes recommendations such as weight reduction, use of light-weight and loose clothing, frequent cleaning and drying of flexural areas, as well as avoidance of hot environments and physical activities that cause friction. In this case, a two-week treatment with topical antimicrobials and corticosteroids showed significant improvement in the patient's lesions, however options for more sustainable treatment must be considered. Promising, emerging therapies for treatment of Hailey-Hailey disease include use of botulinum toxin, laser surgery, dermabrasion, and the opiate antagonist naltrexone. ${ }^{1,11}$

\section{CONCLUSION}

Hailey-Hailey disease, though uncommon, should always be considered in cases of chronic eczematous or scaly annular lesions found at sites of friction, with a positive family history and noted exacerbating factors, such as heat and sweating. This was a case of a 64-year old Filipino female who presented with recurrent, malodorous intertriginous plaques, who had consulted with multiple physicians for her condition with only minimal improvement upon treatment. Prompt diagnosis of such cases is important as patients with Hailey-Hailey disease often present with reduced quality of life due to the recurrent nature of this condition as well 
as its pruritic, painful, and malodorous lesions. Physicians should always assess the patient's family members for similar symptoms, remembering that phenotype and severity can differ even among relatives due to multiple intrinsic genetic and extrinsic factors, as was seen in the varying presentations amongst the different family members of the patient. Clinical presentation, family history, and histopathological evaluation are needed to diagnose Hailey-Hailey disease and additional direct immunofluorescence studies may prove to be confirmatory. This was of significance particularly in this case, as there were overlapping features of pemphigus vulgaris and Hailey-Hailey disease. Treatment should emphasize reducing factors that cause disease exacerbation, though studies on more sustainable forms of treatment should still be conducted to help in the long-term management of these patients. Our patient noted improvement of her lesions with preventive measures and treatment with topical antibiotics and corticosteroids.

\section{Statement of Authorship}

All authors participated in data collection and analysis, and approved the final version submitted.

\section{Author Disclosure}

All authors declared no conflicts of interest.

\section{Funding Source}

No funding support.

\section{REFERENCES}

1. Kang S, Amagai M, Bruckney AL, Enk AH, Margolis DJ, McMichael AJ, et al. Hailey-Hailey Disease. Fitzpatrick's Dermatology, 9th edition. McGraw-Hill Education. 2019, chapter 50, pp. 886-90.

2. Patel VM, Rubins S, Schwartz RA, Septe M, Rubins A. Hailey-Hailey disease: a diagnostic challenge. Cutis. 2019 Mar;103(3):157-9.

3. Laga AC, Haefner HK, Granter SR. Noninfectious Inflammatory Disorders of the Vulva. Diagnostic Gynecologic and Obstetric Pathology, 3rd edition. Elsevier. 2017, pp. 41-2.

4. Philippine Dermatological Society, Health Information System Task Force. Hailey-Hailey Disease Census, 2011-2018.

5. Tansini PB, Boff AL, Weber MB, Bonamigo RR. Familial "benign" pemphigus? Erythroderma and fatal outcome. An Bras Dermatol. 2020 Jan-Feb;95(1):75-7.

6. Van Beek N, Patsatsi A, Gupta Y, Möller S, Freitag M, Lemcke S, et. al. A family with atypical Hailey Hailey disease--is there more to the underlying genetics than ATP2C1? PLoS One. 2015 Apr 2;10(4):e0121253.

7. Deng $\mathrm{H}$, Xiao H. The role of the ATP2C1 gene in Hailey-Hailey disease. Cell Mol Life Sci. 2017 Oct;74(20):3687-3696.

8. Gisondi P, Sampogna F, Annessi G, Girolomoni G, Abeni D. Severe impairment of quality of life in Hailey-Hailey disease. Acta Derm Venereol. 2005;85(2):132-5. doi: 10.1080/00015550410025462. PMID: 15823906.

9. Jain V, Jindal N, Imchen S. Localized Pemphigus Vegetans without Mucosal Involvement. Indian J Dermatol. 2014 Mar;59(2):210.

10. Arbache ST, Nogueira TG, Delgado L, Miyamoto D, Aoki V. Immunofluorescence testing in the diagnosis of autoimmune blistering diseases: overview of 10-year experience. An Bras Dermatol. 2014 Nov-Dec;89(6):885-9.

11. Ben Lagha I, Ashack K, Khachemoune A. Hailey-Hailey Disease: An Update Review with a Focus on Treatment Data. Am J Clin Dermatol. 2020 Feb;21(1):49-68.

\section{The Acta Medica Philippina is now accepting limited advertising for its front and back cover (colored), as well as for available spaces in some of its pages, as appropriate. For inquiries and submission of proposals, please email us at actamedicaphilippina.upm@up.edu.ph}

